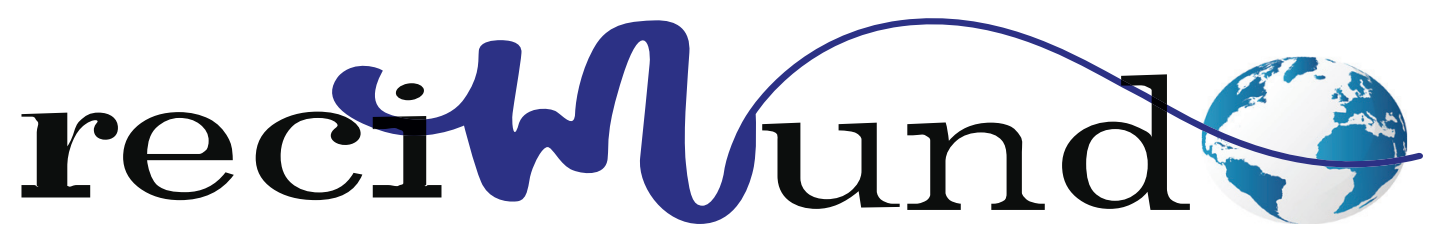

Revista Científica Mundo de la Investigación y el Conocimiento

DOI: 10.26820/recimundo/4.(4).octubre.2020.468-481

URL: http://recimundo.com/index.php/es/article/view/963

EDITORIAL: Saberes del Conocimiento

REVISTA: RECIMUNDO

ISSN: 2588-073X

TIPO DE INVESTIGACIÓN: Artículo de Revisión

CÓDIGO UNESCO: 5605 Legislación y Leyes Nacionales

PAGINAS: 468-481

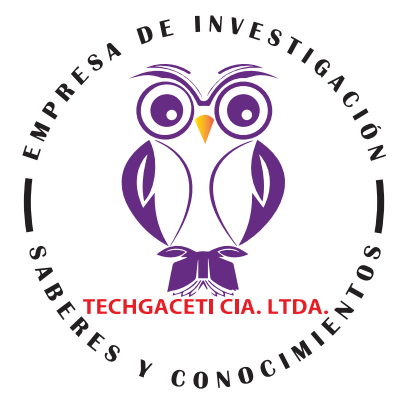

\title{
Comentarios acerca del lavado de activos y el delito previo, especial referencia al COIP
}

Comments about money laundering and prior crime, special reference to the COIP Comentários sobre branqueamento de capitais e crimes anteriores, referência especial ao COIP

Jennifer Katiusca Banchón Cabrera'; Gabriel Yovany Suqui Romero²

RECIBIDO: 10/11/2020 ACEPTADO: 26/11/2020 PUBLICADO: 13/12/2020

1. Estudiante de la Universidad Técnica de Machala; Investigadora Independiente; Machala, Ecuador; jbanchon2@utmachala.edu.ec; (iD) https://orcid.org/0000-0002-0462-0616

2. Máster Universitario en Derecho; Doctor en Jurisprudencia; Abogado de los Tribunales de Justicia de la República; Orientación Investigadora Especialidad En Derecho Penal; Docente Universidad Técnica de Machala; Machala, Ecuador; gsuqui@utmachala.edu.ec; iD https://orcid.org/0000-0002-3704-8193

CORRESPONDENCIA

Gabriel Yovany Suqui Romero

gsuqui@utmachala.edu.ec

Machala, Ecuador

๑) RECIMUNDO; Editorial Saberes del Conocimiento, 2020 


\section{RESUMEN}

La complejidad del delito de lavado de activos se evidencia no sólo en la redacción del tipo penal escogida por el legislador ecuatoriano, sino que también en la figura del delito previo como un elemento constitutivo del delito de lavado de activos. Por lo antes expuesto, el objetivo de esta investigación es delimitar el alcance de la normativa internacional en el ordenamiento jurídico penal ecuatoriano respecto al delito de lavado de activos con énfasis en los lineamientos del delito previo, mediante el análisis sistemático y dogmático de la normativa penal, para comprender la naturaleza e influencia de este delito previo dentro de los procesos penales. Finalmente, se arriba a la conclusión que el tipo penal de lavado de activos, desde la óptica mundial destaca la importancia que se le dio en un primer momento al estar íntimamente arraigado a delitos graves, en especial al narcotráfico, para luego ser considerado un delito autónomo. Con base a lo analizado, el delito previo tiene una incidencia fundamental en el tipo penal del lavado de activos, aunque se visualiza de forma clara que la única referencia o ligadura que en el caso ecuatoriano realiza el legislador es con los bienes y su ilicitud, tal como emana de la redacción del tipo penal.

Palabras clave: Lavado de activos, Delito previo, Código Orgánico Integral Penal, Derecho penal económico.

\section{ABSTRACT}

The complexity of the crime of money laundering is evidenced not only in the wording of the criminal type chosen by the Ecuadorian legislator, but also in the figure of the previous crime as a constitutive element of the crime of money laundering. Due to the aforementioned, the objective of this investigation is to define the scope of the international regulations in the Ecuadorian criminal legal system regarding the crime of money laundering with emphasis on the guidelines of the previous crime, through the systematic and dogmatic analysis of the criminal regulations, to understand the nature and influence of this prior crime within criminal proceedings. Finally, it comes to the conclusion that the criminal type of money laundering, from a global perspective, highlights the importance that was given at first to be intimately rooted in serious crimes, especially drug trafficking, to later be considered a crime autonomous. Based on the analysis, the prior crime has a fundamental impact on the criminal offense of money laundering, although it is clearly seen that the only reference or link that in the Ecuadorian case the legislator makes is with the goods and their illegality, as emanates from the wording of the criminal type.

Keywords: Money laundering, Prior crime, Comprehensive Organic Criminal Code, Economic criminal law.

\section{RESUMO}

A complexidade do crime de branqueamento de capitais é evidenciada não só na redacção do tipo de crime escolhido pelo legislador equatoriano, mas também na figura do crime anterior como elemento constitutivo do crime de branqueamento de capitais. Devido ao acima mencionado, o objectivo desta investigação é definir o âmbito da regulamentação internacional no sistema jurídico penal equatoriano relativamente ao crime de branqueamento de capitais com ênfase nas directrizes do crime anterior, através da análise sistemática e dogmática da regulamentação penal, para compreender a natureza e influência deste crime anterior no processo penal. Finalmente, chega-se à conclusão de que o tipo criminoso de branqueamento de capitais, numa perspectiva global, realça a importância que foi dada no início para estar intimamente enraizado nos crimes graves, especialmente no tráfico de droga, para mais tarde ser considerado um crime autónomo. Com base na análise, o crime anterior tem um impacto fundamental no delito criminal de branqueamento de capitais, embora se veja claramente que a única referência ou ligação que no caso equatoriano o legislador faz é com os bens e a sua ilegalidade, como emana da redacção do tipo criminoso.

Palavras-chave: Branqueamento de capitais, Crime anterior, Código Penal Orgânico Abrangente, Direito Penal Económico. 


\section{Introducción}

Desde su surgimiento en la esfera internacional, el lavado de activos ha atraído la atención de los juristas por dos causas fundamentales. En primer lugar, la complejidad de las conductas que se enmarcan dentro del tipo penal de lavado de activos y la lesión a una multiplicidad de bienes jurídicos (orden socioeconómico, la correcta administración de justicia, entre otros). En segundo lugar, dado a que posee la capacidad de reinversión del dinero proveniente de delitos tanto en el flujo legal de una sociedad, así como en la financiación de más delitos, y aquí radica la importancia de la lucha estatal contra estas prácticas de lavado. Por estos motivos, la creciente preocupación antes señalada no es vaga, sino que tiene por objetivo la delimitación de concepciones fundamentales (entre las que se encuentra la independencia con respecto al delito previo) a la hora del análisis de este delito.

Desde la entrada en vigencia del Código Orgánico Integral Penal (en adelante, COIP) en el año 2014, Ecuador regula el delito de lavado de activos en el art. 317 y siguientes de este cuerpo normativo que se complementa con la Ley Orgánica de Prevención, Detección y Erradicación del Delito de Lavado de Activos y del Financiamiento de Delitos (del año 2016) como normativa conexa.

Dentro de la multiplicidad de aristas dignas de análisis que posee el delito de lavado de activos, es el delito previo uno de los temas más interesantes y problemáticos. Cabe entender que el delito de lavado de activos persigue el ocultamiento de bienes de origen ilícito, por tanto, es primordial abordar el análisis del delito del cual nace el lavado, es decir el delito fuente, toda vez que el lavado de activos es un tipo de conexión que conecta con un hecho penal previo, y que como bien señala MENDOZA LLAMACPONCCA (2019), en un proceso penal se torna indiscutible la necesidad de verificarse la ilicitud de los bienes así como los posteriores actos de legitimación o lavado.

\section{Desarrollo}

\section{Marco normativo internacional de lava- do de activos y referencia al delito previo}

El marco normativo internacional del lavado de activos es bastante amplio, pues, combina instrumentos internacionales con carácter mundial, regional y bilateral, así como pronunciamientos de instituciones no estatales, como corporaciones internacionales -ejemplo, las federaciones o asociaciones bancarias- (Prado Saldarriaga, 2008).

Tiene su surgimiento en el marco de la lucha internacional por controlar ciertos delitos -tráfico de drogas, trata de personas, financiamiento del terrorismo, por ejemplo-, reconociendo que la intención de los grupos criminales es la generación de ganancias (Pinto \& Chevalier, 2001) y su posterior reutilización, siendo necesario, por ende, disimular su origen ilícito. Una muestra de ello es la forma en la que se regula el lavado de activos en la Convención de Viena de 1988, donde es considerado como una mera técnica legislativa para combatir el tráfico ilícito de drogas (Fabián Caparrós, 2007, p. 13). Esto da la pauta que inicialmente la comunidad mundial considere al lavado de activos como una parte del proceso de financiación de las organizaciones delictivas, por lo que este delito se mantenía atado a un numerus clausus reducido. Sin embargo, esta situación iba a cambiar, y la doctrina comenzaría a esbozar criterios que servirían para separar al lavado de activos de otros delitos y de esta manera, considerarlo un delito autónomo, concepción que tiene vital importancia a la hora del estudio del delito previo. De esta manera, la preocupación principal era "desfinanciar" a las organizaciones delictivas, para lo cual, se preveía dotar a los países de una serie de mecanismos para retener estas ganancias e interrumpir el traspaso del dinero desde el 
tráfico de drogas a otras actividades ilícitas (Huayllani Vargas, 2016, p. 24).

Esa preocupación permitió que la actual concepción del lavado de activos se vaya incorporando de a los ordenamientos jurídicos internos de los países que fueron ratificando los distintos instrumentos internacionales, aunque de forma lenta. De igual modo, el lavado de activos se fue erigiendo como un delito autónomo, aunque ligado siempre a la conducta previa que genera la ilicitud de los bienes que se lava o se pretenden lavar. Sobre esta base se desarrollaron con el devenir del tiempo múltiples interpretaciones doctrinales que serán abordadas en el presente trabajo, con especial referencia a aquellas relativas al delito previo.

La internacionalización del delito de lavado de activos conlleva no sólo la introducción en los diferentes ordenamientos jurídicos de los países parte de las convenciones y tratados, sino también la adopción de una serie de mecanismos administrativos y procesales con miras a una homogeneidad de las legislaciones en la lucha contra este fenómeno delictivo (Gómez Iniesta, 1996, p. 18). Parte de la homogeneidad que se buscaba y parece estar lograda en la actualidad, corresponde en gran medida a los elementos normativos y descriptivos del tipo penal, con gran enfoque en los bienes o activos que dentro del análisis gramatical del tipo penal constituyen el objeto material del delito.

\subsection{Convención de Viena}

En el marco del análisis del objeto material del delito, la Convención de Viena de 1988 dio un primer puntapié y definió a los bienes como "los activos de cualquier tipo, corporales o incorporales, muebles o raíces, tangibles o intangibles, y los documen- tos o instrumentos legales que acrediten la propiedad $u$ otros derechos sobre dichos activos"1. Además, define a los "productos" como el origen de los bienes señalados anteriormente (Del Carpio-Delgado, 2015). Lo innovador de la definición separada de "bienes" y "productos" trascendería la esfera de esta Convención para luego recalar en otros instrumentos internacionales de lucha contra el lavado de activos, tales como los Convención de Palermo y la de Mérida. En este primer estadio de la tipificación del delito de lavado de activos se puede apreciar que si bien se persigue el encubrimiento de la verdadera naturaleza de los bienes que son producto de un delito previo, estos bienes deben provenir solamente del tráfico ilícito de drogas y actividades conexas (con base al art. 3 de la Convención de Viena). Por lo que se considera al lavado de activos, solamente como una técnica legislativa (Fabián Caparrós, 2007, p. 13).

\subsection{Las 40 Recomendaciones del GAFI}

Apenas un año después, en 1989, el GAFI (Grupo de Acción Financiera) emite las cuarenta recomendaciones (las cuales tienen sucesivas modificaciones en los 1996, 2001, 2003 y 2012), mismas que buscan un mayor protagonismo de las instituciones financieras y crediticias, promoviendo una cooperación internacional entre este tipo de instituciones (Rodríguez Villar \& Germán Bermejo, 2001, p. 61). En un inicio, en la primer etapa de estas 40 Recomendaciones (desde el año 1990 hasta el 1996) sólo se contenía dentro de los delitos previos aquellos relacionados con el tráfico de drogas, más, este documento tuvo luego modificaciones que pretenden abarcar un espectro más amplio de delitos determinantes -así son llamados los delitos previos en estas recomendaciones-, incluyendo los denominados "delitos graves", con penas mayores

${ }_{1}^{1}$ Artículo 1, literales p y q, Convención de las Naciones Unidas Contra el Tráfico Ilícito de Estupefacientes y Sustancias Psicotrópicas, adoptada en Austria el 19 de diciembre de 1988. 
a un año de prisión (OCDE-GAFI, 2012, p. 35), cambiando la técnica legislativa empleada hasta el momento.

\subsection{Reglamento modelo de la CICAD}

La Comisión Interamericana Contra el Abuso de Drogas (CICAD) bajo pedido de la Asamblea General de la OEA conformó una comisión de expertos para la investigación del fenómeno de lavado de activos. Producto de estas investigaciones surge el Reglamento Modelo (el cual sufrió modificaciones en los años 1997, 1998 y 2005), dentro de los mayores aportes de este Reglamento se encuentra la reafirmación en cuestiones de prevención de financiamiento del terrorismo así como de la autonomía del delito de lavado de activos y del delito previo, y temas referentes a empresas financieras informales. Como desventaja, se puede mencionar que al pertenecer al grupo del soft law, los pronunciamientos allí vertidos no poseen exigibilidad alguna, aunque en la práctica, por la calidad de los aportes, los Estados han ido tomando algunos pasajes y los han incorporado a sus ordenamientos jurídicos, siendo quizás lo más destacado la tipificación autónoma del delito de lavado de activos.

Se puede citar también como avance del Reglamento Modelo, la concepción de la posibilidad de la comisión del delito de lavado de activos por dolo directo, dolo eventual, así como imprudencia (Fabián Caparrós, 2007), esto con base al art. 2.1.

\subsection{Convenio de Estrasburgo}

Este instrumento da un paso elemental al pretender dotar de autonomía al delito de lavado de activos, dada la rentabilidad que genera la comisión de delitos, sean de la naturaleza que sea (Huayllani Vargas, 2016, p. 25). Se da un avance, igualmente, al prohibir que las entidades financieras recurran al secreto bancario cuando la información sea requerida por solicitudes de auxilio judicial en investigaciones por lavado de activos
(Zaragoza Aguado, 2007), esto con base en los arts. 4.1 y 18.7 de este Convenio. Esta característica tiene como fundamento la idea de que las instituciones deben cooperar con la administración de justicia, concepto que luego se vería reforzado con las recomendaciones emitidas por juntas bancarias nacionales así como asociaciones internacionales, como en el caso del Comité de Supervisión Bancaria de Basilea.

Como bien resalta Fabián Caparrós (2007), el Convenio de Estrasburgo (1990) deja atrás la dependencia del lavado de activos con respecto al delito previo, estableciendo en su art. 6.1 la obligación de los Estados parte a penalizar las conductas relacionadas con el lavado de activos, siendo relevante la existencia de una ganancia ilícita y no la característica concreta del delito previo, dotando de autonomía al lavado de activos, rompiendo con el esquema de su accesoriedad a la infracción previa.

\subsection{Convención de Palermo}

La Convención de Palermo (2000), da un paso más adelante en comparación a sus predecesores y aumenta o amplía el campo de los delitos previos, pasando tipificar el encubrimiento de bienes surgidos de los delitos conexos al tráfico ilícito de sustancias estupefacientes, a los bienes que tengan como origen en "un delito" (art. 6). Con esta fórmula, se da paso al estudio del delito previo o determinante, que como destaca Del Carpio- Delgado (2015) será "todo delito del que se derive un producto que pueda pasar a constituir materia de un delito constitutivo de blanqueo de capitales" (p. 672). Otro avance se encuentra en el numeral 2 del art. 6 de esta Convención, donde se insta a los Estados parte a ampliar el abanico posible de delitos previos.

Según destaca Fabián Caparrós (2007), esta Convención, tiene como intención el procesamiento por lavado a los sujetos activos del delito previo, dejando claro que 
son dos conductas diferentes, y que cuando se trate de autolavado o autoblanqueo, con base a lo establecido en su art. 6.2.e, mismo que señala que únicamente frente a la imposibilidad del Derecho interno del Estado parte, "podrá disponerse que los delitos tipificados en el párrafo (1) del presente artículo no se aplicarán a las personas que hayan cometido el delito determinante" ( $p$. 31 ), entendiéndose el delito determinante como delito previo o fuente.

\subsection{Convención de Mérida}

Al igual que la antecesora Convención de Palermo, la Convención de las Naciones Unidas contra la Corrupción de 2003 (Convención de Mérida), mantiene la intención de ampliar el horizonte de los delitos previos (art. 23.2.a), así como también mantiene la cláusula prevista en la Convención de Palermo, la que señala que en el caso de que la comisión de la conducta que da lugar a los bienes que se lavaron o se pretendieron lavar haya tenido lugar en otra jurisdicción ajena a la del Estado parte que pretende procesar por lavado de activos, será necesario que dichas conductas se encuentren tipificadas como delito en este país de origen (art. 23.2.c).

Si bien la idea central es el combate a la corrupción, sucesivos pasajes de esta Convención aportan a la normativización internacional de lucha contra el lavado de activos, destacándose la necesidad de la prevención administrativa con las unidades de análisis financiero -en el Ecuador, la UAFE- (art. 14); la penalización del delito de lavado de activos de manera autónoma o independiente al lugar de la comisión del delito previo (art. 23.2) así como la impor- tancia del castigo al blanqueador o lavador con independencia de su responsabilidad en la comisión o participación en el delito previo (art. 23.1.a.ii). Del Carpio-Delgado (2015) señala también como un aporte importante que "para configurar el delito es esencial que el sujeto haya recibido los bienes, es decir, debe haber una «recepción» de los mismos" (p. 709).

\subsection{Conclusiones preliminares con base al marco normativo internacional de lavado de activos y referencia al delito previo}

De lo advertido ut supra se percibe que, como destaca Mendoza Llamacponcca (2019), los instrumentos internacionales de lucha contra el lavado de activos "obligan a sus Estados integrantes a prevenir el blanqueo de capitales procedentes del delito previo, mas no así de los derivados de un «origen ilícito» en sentido general" (p. 313). La importancia de esta evolución es dada por el carácter internacional del delito de lavado de activos, cuyo surgimiento es en el seno internacional y el acuerdo de la necesidad de los países por poner fin a una práctica con marcado carácter transnacional, tanto por la esencia del lavado como por los delitos que dan surgimiento a los bienes o activos que se lavan, como, por ejemplo, es el tráfico ilícito de drogas.

El rompimiento de esta dependencia entre el delito de lavado de activos y la persecución del tráfico de drogas se da de manera clara en el Reglamento Modelo de la Cl$\mathrm{CAD}$, donde se aprecia que la intención de la comisión de expertos es que los países miembros incluyan una gama más amplia de delitos previos², así como la necesidad de una persecución autónoma e indepen-

\footnotetext{
${ }^{2}$ «Concediéndole relevancia en tanto los bienes procedieran de "actividades delictivas graves”, esto es (ex art. 1), aquellos actos criminales "determinados por la legislación de cada Estado, incluyendo el tráfico ilícito; acciones relacionadas con el terrorismo y la financiación del terrorismo, actos terroristas y organizaciones terroristas; tráfico ilícito de armas; desvío de sustancias químicas; tráfico ilícito de humanos y tráfico de órganos humanos; prostitución; secuestro; extorsión, corrupción y fraude"». En Fabián Caparrós, E. (2007). "Antecedentes: Evolución de las iniciativas internacionales. Efectos del lavado de dinero. Bien jurídico tutelado. Fenomenología del lavado de dinero", en AA.VV., E. Fabián Caparrós, I. Blanco Cordero, V. Prado Saldarriaga, J. Zaragoza Aguado (eds.), Combate del Lavado de Activos desde el Sistema Judicial, (pp. 9-96), CICAD, Banco Interamericano de Desarrollo, p. 29.
} 
diente con respecto al enjuiciamiento de este ilícito previo (art. 4). De este modo se da más atención al delito de lavado de activos así como al delito previo, en un primer momento con carácter internacional, para luego con esta estimulación y el posterior compromiso de los Estados parte, darse el traslado a los ordenamientos jurídicos internos.

Juega sin lugar a dudas un papel muy importante, la clasificación de instrumentos de soft law y de hard law a la hora de valorar de qué modo y con qué diligencia los Estados han introducido las modificaciones solicitadas o recomendadas en estos cuerpos normativos, aunque por el análisis efectuado, los Estados en su mayoría han estado abiertos a tales modificaciones. Como se podrá apreciar en apartados siguientes, Ecuador a partir de la entrada en vigencia del COIP, recoge la mayoría de las recomendaciones y conceptos dados en los convenios y demás cuerpos normativos, tanto vinculantes como no vinculantes, en especial lo referente a la independencia y autonomía espacial del delito de lavado de activos y los delitos previos así como una amplia gama de verbos rectores.

\section{Consideraciones respecto al delito pre- vio}

En la actualidad, es indudable la importancia que se ha dado al delito previo, tanto desde la óptica internacional como en la esfera nacional. Sin embargo, muchas veces se ha dejado de lado la discusión del delito previo bajo pretexto de que para procesar al lavado de activos no es necesaria la determinación del origen delictivo de los bienes. La esencia del lavado de activos, como se ha apreciado hasta el momento, es el origen ilícito de ciertos bienes o activos y su posterior ingreso o pretensión de ingreso dentro del tráfico económico legal (Martín Sagrado, 2018).

La necesidad de la acreditación del delito previo queda supeditada, según cierta parte de la doctrina, a que esto sea parte del tipo penal de lavado de activos (Gálvez Villegas, 2014) ${ }^{3}$, mientras que otros indican que de ser incluido el delito previo a la estructura típica "simplemente el tipo penal de lavado resultaría inaplicable" (Gálvez Villegas, 2014) ${ }^{4}$. Por esto, muchas veces se deja de lado la investigación del delito previo que acredite la ilicitud de los bienes que son sujetos a lavado, y se fundamenta la persecución penal por el hecho de no encontrarse sustento a movimientos inusuales de dinero. Si bien se puede dar un concurso, entre una persona que comete el delito previo y posteriormente realiza actos tendientes al ocultamiento o disimule de los bienes obtenidos por las actividades ilícitas previas, la necesidad de una autonomía sustantiva y en sede procesal se da por varias razones, entre las que destaca la lentitud en la investigación y juzgamiento del delito previo, sumado a la diferencia que se puede suscitar con respecto a los bienes jurídicos atentados en las diferentes conductas (Gutiérrez Chávez, 2019).

La independencia de la tipificación del delito de lavado de activos se sustenta en el hecho de que este delito lesiona bienes jurídicos que pueden ser diferentes a los ya lesionados por el delito previo (Durrieu Figueroa, 2012), aunque la doctrina en la actualidad ha desplazado la importancia de la valoración de los bienes jurídicos. De igual manera, la autonomía del delito de lavado de activos permite el llamado autolavado, donde el autor del delito previo es a su vez el autor del delito de lavado de activos (Mendoza Llamacponcca, 2019).

\footnotetext{
${ }^{3}$ En este sentido, el autor establece que "no hace falta acreditar un delito previo sino únicamente establecer una conexión entre la acción de lavado y las actividades criminales" p. 91.

"En similar sentido, Mendoza Llamacponcca, F. (2019). El tipo base del delito de lavado de activos en el Perú (arts. 1, 2 y 3 del Decreto Legislativo $n^{\circ}$ 1106). Apuntes desde el Derecho penal español. Tesis doctoral, Universidad de Salamanca, p. 317.
} 
Con respecto a la autonomía del delito de lavado de activos y su reflejo en las penas establecidas, cabe trasladar el análisis de Aránguez Sánchez al caso ecuatoriano desde el caso español, donde este autor manifiesta que "la total autonomía (...) respecto al delito previo se aprecia nítidamente en la pena prevista para el blanqueador, la cual es determinada por el legislador con independencia de la establecida para el delito del que provienen los bienes" (Aránguez Sánchez, 2000, p. 296).

\section{Autonomía o dependencia sustantiva y procesal del delito de lavado de activos con respecto al delito previo}

El delito de lavado de activos nace muy ligado a ciertos delitos previos, generalmente asociado al tráfico de sustancias estupefacientes, pasando luego a un grupo un poco más amplio de delitos graves (Chavarría Cedillo, 2016) pero tan apegado a ellos al punto de ser contemplado en los inicios como una mera técnica legislativa. Este surgimiento ha condicionado la forma en la que las legislaciones nacionales han tratado al delito de lavado de activos, donde cabe analizar la importancia que se le brinda al delito previo tanto en materia sustantiva como procesal. Esta especificación es no menor, dado que de ella dependerá el tratamiento procesal que se le dé al lavado de activos. Por medio de la doctrina se han erigido dos pensamientos antagónicos, donde el delito previo y su apreciación juegan papeles totalmente diferentes con respecto al procesamiento del lavado de activos.

Existe, por otra parte, cierto sector de la doctrina que muestra una negativa frente a la concepción del lavado de activos como un delito en sí, sino, más bien, como un injusto relacionado al delito previo (Suárez González, 1996). Si bien es cierto, los doctrinarios en su mayoría coinciden con respecto a la autonomía procesal, sin embargo, no se verifica la misma uniformidad de criterios con respecto a la autonomía sustantiva, llegando algunos a señalar que no es posible una separación del delito de lavado de activos con respecto al delito previo y que la autonomía sustantiva atenta contra ciertos principios constitucionales (Pariona Arana, 2014).

\subsection{La teoría de la autonomía procesal del delito de lavado de activos}

Esta teoría o pensamiento centra su posición en la autonomía del delito previo en relación al delito de lavado de activos en sede procesal. Así, se puede apreciar que al tratar de manera autónoma el delito previo y el lavado de activos, se podría procesar al autor del delito previo por tal delito, y de igual manera, procesarlo por el delito de lavado de activos (Franzini-Battle, 1995).

La concepción del delito de lavado de activos como un delito autónomo con respecto al delito previo suscita más discusiones en el ámbito procesal que en lo referente a la redacción del tipo penal. Esto es así dado que para algunos autores, la ligadura del tipo de lavado de activos al delito previo llevaría a una inutilidad del tipo penal, ineficacia de la administración de justicia y a su vez, una burla tanto a las expectativas sociales como a la política criminal que lleva a la creación del delito de lavado de activos ${ }^{5}$.

De modo crítico, se puede establecer que dentro del actuar lógico que el delincuente, luego de generar un ingreso proveniente de actividades ilícitas, busque asegurar su goce o disfrute, siendo por tanto una continuación inherente al delito previo. Con base a este razonamiento, el autoblanqueo o autolavado se erige como una doble penalización dentro de una misma conducta (conti-

${ }^{5}$ Gálvez Villegas, T. (2016). Autonomía del delito de lavado de activos. Cosa decidida y cosa juzgada. Lima: Ed. Ideas, pp. 195-196. 
nuación del delito previo), por tal motivo, se considera que el delito de lavado de activos depende de la previa comisión de otro delito (Martínez-Buján Pérez, 2013).

La crítica que da lugar a esta concepción de autonomía procesal nace con la legitimación de las investigaciones por presunto lavado de activos, cuando aún no se tenía la seguridad sobre la ilicitud de los bienes supuestamente lavados, de igual manera se sucedían cuestionamientos acerca de la no acreditación judicial de la ilicitud de los bienes materia del lavado (Pariona Arana, 2014). Esto da a entender que, dentro de un sistema democrático de derechos y justicia, más aún con una Constitución garantista, estos aspectos no pueden darse por sentados y continuar con el procesamiento, sino investigarse la real proveniencia de estos bienes y de ser ilícitos, procesar a los responsables por el delito de lavado de activos.

Centrando este análisis en la normativa ecuatoriana, el COIP señala en su art. 317 con referencia al catálogo de conductas de lavado de activos, que "Estos delitos son considerados como autónomos de otros cometidos dentro o fuera del país, sin perjuicio de los casos en que tenga lugar la acumulación de acciones o penas. Esto no exime a la Fiscalía de su obligación de investigar el origen ilícito de los activos objeto del delito". De esta manera, el legislador ecuatoriano deja claro que en su concepción, el delito de lavado de activos tiene una autonomía tanto material ${ }^{6}$ como procesal.

\subsection{La teoría de la autonomía sustantiva del delito de lavado de activos}

Una vez aclarada superficialmente el pensamiento que sustenta la autonomía procesal del delito de lavado de activos con respecto al delito previo, surge la necesidad de establecer los lineamientos que la doctrina ha expuesto con respecto a la autonomía sustantiva. De manera preliminar se puede indicar que la autonomía sustantiva suscita mayores controversias que la autonomía procesal, pues, asumir esta postura resultaría en la creación de un tipo penal que no haga referencia a la cualidad ilícita de los bienes o activos.

Con la situación plasmada en el presente trabajo, hasta el momento no sería factible quitar un elemento de tal trascendencia para el procesamiento del lavado de activos, como es el carácter ilícito de los bienes que se introducen al tráfico económico legal. Tal como desataca Prado Saldarriaga (2008) para el caso peruano, en Ecuador, de igual manera, el legislador ha "ligado" al tipo penal de lavado de activos con la procedencia de los activos o bienes, es decir, con el delito previo. Se puede acotar a esto que la "ligadura" a un delito previo es la esencia del lavado de activos, la persecución de bienes o activos que se generan por la comisión de otros delitos, y que, a causa de esto, en un primer momento el lavado de activos era considerado una técnica legislativa en pro de la persecución de otros delitos. Si se alcanzó en la actualidad cierta independencia, debe ser ésta concebida dentro de los marcos razonables de una dependencia inherente a la condición propia del encubrimiento del lavado.

Con referencia al marco normativo ecuatoriano, el art. 317 del COIP requiere como elementos descriptivos, que los bienes o activos objeto del delito deben ser de "origen" o procedencia ilícita", por tanto, da a entender que subyace o se antepone una actividad delictiva que da lugar a dichos bienes o activos.

${ }^{6}$ De manera similar analiza la situación contemplada en el Código Penal peruano el autor Gálvez Villegas, T. (2016). "Autonomía del delito...", p. 199. 


\subsection{El delito previo como elemento del tipo penal de lavado de activos}

Dentro de esta temática se concibe que para que la conducta del sujeto activo encuadre en lo señalado en el tipo penal de lavado de activos, es necesario acreditar la ilicitud de los bienes que son lavados. Esto conlleva una traba, en el sentido de que para procesar a una persona por lavado de activos exista de manera previa una sentencia ejecutoriada que establezca que los bienes fueron producto de actividades ilícitas. Para algunos autores, como Puyana Ramos (1995), esta suerte de limitación -la exigencia de sentencia ejecutoriadaviolenta el principio de libertad probatoria, "conforme al cual cualquier circunstancia del delito puede ser probada, con cualquier medio de prueba" (p. 11), con las limitaciones que establece la Constitución y la ley.

En Ecuador, el tipo penal de lavado de activos (art. 317) contiene la cláusula "Estos delitos son considerados como autónomos de otros cometidos dentro o fuera del país, sin perjuicio de los casos en que tenga lugar la acumulación de acciones o penas. Esto no exime a la Fiscalía de su obligación de investigar el origen ilícito de los activos objeto del delito", lo que da a entender que el legislador ecuatoriano comprende que en ciertos casos, puede darse primero el procesamiento por el delito de lavado de activos, y luego la investigación y el proceso por el o los delitos previos que dan lugar a los bienes o activos ilícitos. De ahí que, en el caso ecuatoriano así como otros países de la región, como en el caso brasilero, analizado por Callegari (2003) o el caso colombiano, estudiado por Bernate Ochoa (2006), la teoría que pondera la necesidad de una condena por el delito previo de manera anticipada al proceso de investigación y juzgamiento de lavado de activos, no sería válida.

\section{El delito de lavado de activos y el delito previo}

4.1. El delito de lavado de activos en el COIP

La redacción del art. 317 señala elementos vitales a la hora de proyectar un análisis sobre las condiciones que, a entender del legislador ecuatoriano, dan lugar al delito de lavado de activos, así como su relación con el delito previo.

En primer lugar, cabe destacar que, como parte de la redacción, en tres de los seis numerales que desglosan las diferentes conductas por las cuales se encuadra la conducta del sujeto activo al tipo penal, se encuentra la frase "activos de origen ilícito" o en el caso del numeral sexto, "dinero de procedencia ilícita". Esto es compatible con lo que la doctrina señala como presunción de delito previo o fuente, inclinando la percepción por una autonomía procesal y no sustantiva.

Con base al análisis gramatical del tipo penal, en el presente subapartado se analizarán las categorías más importantes, siempre tomando como referencia el art. 317 del COIP.

\subsubsection{Sujeto activo}

El sujeto activo dentro del delito de lavado de activos es en primera instancia monosubjetivo, pues, este delito puede ser cometido como mínimo por un solo sujeto o individuo (Vega Arrieta, 2016), aunque esta clasificación no es exclusiva, dado que puede ser también cometido por dos o más personas. También es indeterminado, dado que no le asigna ninguna calidad necesaria al sujeto activo, indicando simplemente "la persona". Esto queda de lado cuando al momento de clasificar las agravantes, el legislador ecuatoriano ha ponderado que es necesario aumentar la punición en los casos donde se abuse "del sistema financiero o de seguros; instituciones públicas o dignidades; o, en el desempeño de cargos

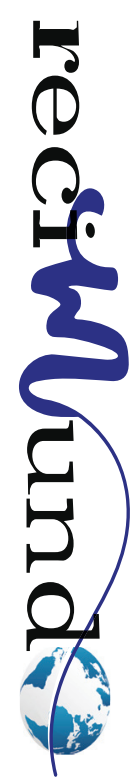


directivos, funciones o empleos en dichos sistemas" (art. 317.2.c) para la comisión de este delito, situación que también se ve en el apartado 3.c de este mismo artículo. Por lo que, en estos últimos casos, el sujeto activo sería cualificado, dado que requiere de una calificación determinada, como la calidad de funcionario o directivo de instituciones financieras.

\subsubsection{Sujeto pasivo}

Por su parte, el sujeto pasivo ha tenido diferentes apreciaciones. Sin lugar a dudas, la gran variación en las concepciones del bien jurídico hace variar también la percepción de quién es el afectado principal derivado de este delito. Por tanto, se hará referencia directa a la administración de justicia así como a la sociedad en su conjunto, dado que se atenta también contra el orden socioeconómico.

\subsubsection{Bien jurídico protegido}

Como se indicó en el apartado anterior, el bien jurídico protegido o tutelado ha suscitado ciertos debates que en la actualidad aún no han sido zanjados. Por un lado, hay un consenso acerca de la pluriofensividad del delito, ya que atenta contra dos o más valores jurídicos (Bustos Ramírez, 1986). Por otro lado, algunos autores establecen que el bien jurídico es la administración de justicia (Blanco Cordero, 1997), dado que la esencia del delito de lavado de activos es el ocultamiento de bienes y activos obtenidos de modo ilícito; pero para otros autores el bien jurídico es el sistema socioeconómico, dentro del cual a decir de Durrieu Figueroa (2011), forman parte ciertas organizaciones internacionales como la OEA, el FMI, BID así como el GAFI. Por último, también es importante señalar que existe cierta postura doctrinaria -Barton, Hinterseer y Zaragoza Aguado, entre otros, destacados por Durrieu Figueroa (2011) - que indica sostienen que en realidad lo que se atenta no es otra cosa que a la seguridad o soberanía y la democracia de los Estados, con fundamento en que el lavado de dinero tiene relación estrecha con la delincuencia organizada y estas organizaciones ponen en peligro estos bienes jurídicos.

En el caso ecuatoriano, de un análisis sistemático, se puede colegir que dado a la ubicación del tipo penal en el COIP como delito económico (Sección 8a, contenido dentro del Capítulo 5 "Delitos contra la responsabilidad ciudadana"), se trata de delitos que afectan al orden económico y que conllevan una afectación a la responsabilidad ciudadana.

\subsubsection{Objeto material del delito}

Con respecto al objeto material del delito, es decir, "la sustancia física o abstracta sobre la cual recae la conducta del sujeto activo" (Vega Arrieta, 2016, p. 60), se vislumbra que se trata de un objeto material real. En el delito de lavado de activos, este objeto lo componen los bienes o activos que se pretende ingresar en el tráfico económico legal. Esta concepción es muy amplia, pero sirve de técnica para que el legislador abarque un gran número de bienes o activos, en sí, objetos o derechos que poseen una cualidad monetaria. En el caso ecuatoriano, el art. 317 menciona "activos" y "dinero".

\subsubsection{Verbos rectores}

Con respecto a los verbos rectores, es decir, aquellos que describen la conducta o el actuar del sujeto activo, en el caso del delito de lavado de activos, tanto desde las recomendaciones, así como los instrumentos internacionales, se ha dado la pauta a un gran número de verbos rectores, mismos que denotan la gran amplitud de las conductas de los sujetos activos de este delito. En el caso ecuatoriano, en el art. 317 se citan los siguientes verbos: "Tenga, adquiera, transfiera, posea, administre, utilice, mantenga, resguarde, entregue, transporte, convierta o se beneficie" (numeral 1), "oculte, disimu- 
le o impida" (numeral 2), "preste" (numeral 3), "organice, gestione, asesore, participe o financie" (numeral 4), "realice" (numeral 5) e, "ingrese o egrese" (numeral 6).

\subsubsection{Elementos normativos y descriptivos}

Como se apreció en cuanto al marco normativo internacional del lavado de activos, el mayor aporte mundial versó en lo referente a la definición de ciertos conceptos como los bienes o activos. En particular, dentro del lavado de activos como los bienes que pueden surgir de actividades delictivas son múltiples, y como de manera obvia, el legislador no puede citar uno por uno dichos bienes que pueden nacer de tales actividades, a empleado la técnica de una denominación genérica de "activos" para evitar, por tanto, un abuso de los elementos descriptivos.

De una revisión a la normativa extrapenal ecuatoriana referente al lavado de activos (Ley Orgánica de Prevención, Detección y Erradicación del Delito de Lavado de Activos y del Financiamiento de Delitos), se verifica que ésta no posee definiciones de elementos que estén presentes en el tipo penal del art. 317. Sin perjuicio de esto, es una ley que aclara procedimientos, atribuciones y responsabilidades de organismos estatales como la Junta de Política y Regulación Monetaria y Financiera, así como la UAFE (Unidad de Análisis Financiero y Económico).

\subsubsection{Rango penológico}

Dentro del art. 317 se encuentra contenido un listado de tres grados penológicos diferentes, mismos que varían con base a los montos de los activos objeto del delito: a) con pena de uno a tres años si el monto no supera los cien salarios básicos unificados del trabajador en general (SBU), b) con pena de siete a diez años cuando el monto de los activos objeto del delito sea igual o superior a cien SBU, y, c) con pena privativa de libertad de diez a trece años cuando el monto de los activos objeto del delito supere los doscientos SBU. Además de esta clasificación basada en una condición objetiva de punibilidad (como son los montos de dinero), estos rangos penológicos también "funcionan" con base al modus operandi del lavado, es decir, si se utiliza una asociación para delinquir utilizando instituciones del sistema financiero o de seguros, instituciones públicas o dignidades, o, en el desempeño de cargos directivos, funciones o empleos en dicho sistema financiero. De igual manera, se señala que la pena se agravará cuando el sujeto activo del delito de lavado de activos ha sido cometido utilizando instituciones públicas, o dignidades, cargos o empleos públicos, así como si se sirve de organizaciones delictivas (delincuencia organizada) o de la constitución de sociedades o empresas, o de la utilización de las que se encuentren legalmente constituidas.

\section{Metodología}

Para la realización del presente trabajo se ha acudido a la revisión bibliográfica y documental, recabadas en motores de búsqueda electrónicos, así como normativa convencional y nacional vigente, para luego aplicar los métodos analítico, exegético y comparativo.

\section{Conclusiones}

Del análisis efectuado al tipo penal de lavado de activos, en primer lugar y desde la óptica mundial se destaca la importancia que se le dio en un primer momento al estar íntimamente arraigado a delitos graves, en especial, al narcotráfico. Luego, con el devenir del tiempo, se aprecia un traslado hacia otros delitos, y por último, se amplía y se concibe como un delito totalmente autónomo y que tiene como único pero complejo requisito, la proveniencia ilícita de los bienes que se lavan o se pretenden lavar. De este modo, la autonomía del delito se consolida en la vía internacional para luego

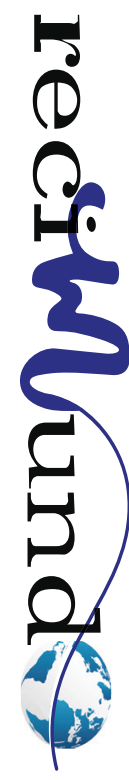


alcanzar los ordenamientos jurídicos nacionales.

Es interesante, además de la reacción penal que buscan los cuerpos normativos internacionales (sean estos vinculantes o no), destacar que uno de los puntos principales también radica en la colaboración internacional, así como la responsabilidad de las instituciones financieras y bancarias en la búsqueda de transparencia y control del movimiento de cantidades inusuales de dinero. De este modo, no solo se previene que se cometa este delito sino también, se busca una confianza en el sistema financiero. Por su parte, la anticipada complejidad de la ilicitud de los bienes, deriva la discusión al grado de importancia que conlleva el delito previo en cuanto al tipo penal de lavado de activos, aunque se dejó claro que la única referencia o ligadura que, en el caso ecuatoriano, realiza el legislador, es con los bienes y su ilicitud, tal como emana de la redacción del tipo penal.

Así mismo, la complejidad del delito se ve reflejada en el tipo penal con una estructura particular, donde en primer lugar se observan una serie de verbos rectores, agrupados por tipos o formas de lavado, para luego incluir ciertos preceptos como la autonomía del lavado de activos, tanto con respecto a otros delitos, así como a la territorialidad de los mismos. Luego, se clasifican los rangos penológicos en atención a la afectación de los bienes jurídicos, así como las posiciones que ostenta el sujeto activo o si se sirve de entidades financieras o empresas.

Capítulo aparte necesitaría la discusión del bien jurídico, donde una vez más se refleja la nocividad del delito, así como su versatilidad. El orden socioeconómico, la administración de justicia, así como la responsabilidad ciudadana son bienes jurídicos puestos en peligro, así lo consideran los doctrinarios citados en el presente trabajo.

\section{Bibliografía}

Aranguez Sánchez, C. (2000). El delito de blanqueo de capitales. Barcelona: Marcial Pons.

Bustos Ramírez, J. (1986). Los bienes jurídicos colectivos. Revista de la Facultad Complutense, vol. 11 , pp. 147-164.

Blanco Cordero, I. (1997). El delito de blanqueo de capitales. Pamplona: Aranzadi.

Chavarría Cedillo, S. (2016). La normatividad internacional en materia de lavado de dinero y su influencia en el sistema jurídico mexicano. Pluralidad y Consenso, vol. 3, núm. 3, pp. 58-63. Disponible en http://revista.ibd.senado.gob.mx/index.php/PluralidadyConsenso/article/view/161/161

Código Orgánico Integral Penal. (2014). Asamblea Nacional del Ecuador. Disponible en https:// www.defensa.gob.ec/wp-content/uploads/downloads/2018/03/COIP_feb2018.pdf

Del Carpio-Delgado, J. (2015). La normativa internacional del blanqueo de capitales: análisis de su implementación en las legislaciones nacionales, España y Perú como caso de estudio. Estudios penales y criminológicos, vol. 35, pp. 657-731.

Durrieu Figueroa, R. (2011). El Bien Jurídico Protegido en el Delito de Lavado de Dinero. Revista del Colegio de Abogados de Buenos Aires, tomo 1, núm. 1, pp. 8-28. Disponible en http://www.colabogados. org. ar/larevista/articulo. php?origen $=\&$ i$d=125 \&$ idrevista $=13$

Fabián Caparrós, E. (2007). "Antecedentes: Evolución de las iniciativas internacionales. Efectos del lavado de dinero. Bien jurídico tutelado. Fenomenología del lavado de dinero", en AA.VV., E. Fabián Caparrós, I. Blanco Cordero, V. Prado Saldarriaga, J. Zaragoza Aguado (eds.), Combate del Lavado de Activos desde el Sistema Judicial, (pp. 9-96), CICAD, Banco Interamericano de DesarroIlo. Disponible en http://www.cicad.oas.org/Lavado_Activos/esp/Documentos/OEA\%20CICAD\%20 LIBRO\%204\%20EDICION.pdf

Franzini-Battle, R. (1995). El delito de lavado de dinero (blanqueo de capitales). Nueva York: Ed. ClCAD.

Gálvez Villegas, T. (2014). El delito de lavado de activos: Criterios sustantivos y procesales. Análisis del Decreto Legislativo № 1106. Lima: Instituto Pacífico.

Gálvez Villegas, T. (2016). Autonomía del delito de lavado de activos. Cosa decidida y cosa juzgada. Lima: Ed. Ideas.

Gómez Iniesta, D. (1996). El delito de blanqueo de capitales en Derecho Español. Barcelona: CEDECS. 
Gutiérrez Chávez, N. (2019). Estándar probatorio en el delito de lavado de activos y su incidencia en el debido proceso respecto a la presunción de inocencia del procesado. Tesis de Máster, Universidad Andina Simón Bolívar, Sede Ecuador. Disponible en http://repositorio.uasb.edu.ec/bitstream/10644/7230/1/T3125-MDPE-Gutierrez-Estandar.pdf

Huayllani Vargas, H. (2016). El delito previo en el delito de lavado de activos. Tesis de Máster, Pontificia Universidad Católica del Perú. Disponible en http://tesis.pucp.edu.pe/repositorio/bitstream/ handle/20.500.12404/7377/HUAYLLANI_VARGAS_HUBER_EL_DELITO.pdf?sequence $=1$ \&isAllowed=y

Ley Orgánica de Prevención, Detección y Erradicación del Delito de Lavado de Activos y del Financiamiento de Delitos. (2016). Asamblea Nacional del Ecuador. Disponible en https://www.cfn.fin. ec/wp-content/uploads/2018/03/Ley-Orga\%CC\%81nica-de-Prevencio\%CC\%81n-de-Lavado-de-Activos-y-del-Financiamiento-de-Delitos. pdf

Martín Sagrado, O. (2018). La determinación del bien jurídico protegido por el delito de blanqueo de capitales y el autoblanqueo. Un debate que no cesa. Boletín del Ministerio de Justicia, núm. 2206, p. 1-37.

Martínez-Buján Pérez, C. (2013). Derecho penal económico y de la Empresa, Parte especial. Valencia: Tirant lo Blanch.

Mendoza Llamacponcca, F. (2019). El tipo base del delito de lavado de activos en el Perú (arts. 1,2 y 3 del Decreto Legislativo $\left.n^{\circ} 1106\right)$. Apuntes desde el Derecho penal español. Tesis doctoral, Universidad de Salamanca. Disponible en https:// gredos.usal.es/bitstream/handle/10366/133000/ DDAFP_LlamacponccaMendozaFN_BaseDelito. pdf;jsessionid=0863BA5C2027EC0609837B90ED080572? sequence $=1$
OCDE-GAFI. (2012). Recomendaciones del GAFI. Disponible en https://www.fatf-gafi.org/media/fatf/ documents/recommendations/pdfs/FATF-40-Rec2012-Spanish.pdf

Pariona Arana, R. (2013). Consideraciones críticas sobre la llamada «autonomía» del delito de lavado de activos. Disponible en http://perso.unifr.ch/derechopenal/assets/files/anuario/an_2013_12.pdf

Pinto R., Chevalier, O. (2001). El delito de lavado de activos como delito autónomo. CICAD/OAS. Disponible en http://www.cicad.oas.org/Lavado_Activos/esp/GupoExpertos/documentos\%2020012005/El\%20delito\%20de\%20lavado\%20de\%20 activos\%20como\%20delito\%20autonomo.doc

Prado Saldarriaga, V. (2008). El delito de lavado de activos en el Perú. Disponible en http:// perso.unifr.ch/derechopenal/assets/files/articulos/a_20080526_63.pdf

Puyana Ramos, G. (1995). El sector financiero y la lucha contra el lavado de activos. Boletín Jurídico Financiero. Bogotá: Asobancaria.

Rodríguez Villar, P., Germán Bermejo, M. (2001). Prevención del lavado de dinero en el sector financiero. Buenos Aires: ADHOC.

Suárez González, C. (1996). "Blanqueo de capitales y merecimiento de pena: consideraciones críticas a la luz de la legislación española", en Cuadernos de Política Criminal (CPC). Madrid: Dykinson.

Vega Arrieta, H. (2016). El análisis gramatical del tipo penal. Revista Justicia, núm. 29, pp. 53-71.

Zaragoza Aguado, J. (2007). "La Nueva regulación del Decomiso de Bienes en el Código Penal y en el Derecho Comparado.", en AA.VV., E. Fabián Caparrós, I. Blanco Cordero, V. Prado Saldarriaga, J. Zaragoza Aguado (eds.), Combate del Lavado de Activos desde el Sistema Judicial, (pp. 333417), CICAD, Banco Interamericano de DesarroIlo. Disponible en http://www.cicad.oas.org/Lavado_Activos/esp/Documentos/OEA\%20CICAD\%20 LIBRO\%204\%20EDICION.pdf

\section{CITAR ESTE ARTICULO:}

Banchón Cabrera, J. K., \& Suqui Romero, G. Y. (2020). Comentarios acerca del lavado de activos y el delito previo, especial referencia al COIP. RECIMUNDO, 4(4), 468-481. https://doi.org/10.26820/recimundo/4.(4).octubre.2020.468-481 